\title{
Numerical Investigation of Installed Jet Noise Sensitivity to Lift and Wing/Engine Positioning
}

\author{
M. Angelino*, M. A. Moratilla-Vega ${ }^{\dagger}$, A. Howlett ${ }^{\ddagger}$, H. Xia ${ }^{\S}$ and G. J. Page ${ }^{\mathbb{I}}$ \\ Department of Aeronautical and Automotive Engineering, Loughborough University, \\ Loughborough, Leicestershire, LE11 3TU, United Kingdom
}

\begin{abstract}
As the turbofan engines of modern transport aircraft have increasingly larger bypass ratios, by necessity to avoid longer undercarriage, the engine must be installed closer to the wing. This then has the potential of interaction between the jet flow and a deployed flap. This interaction can be an important noise source when the high-lift systems are deployed, as at approach and take-off. Investigating the parameters that have a strong influence on the installation noise penalty can help in identifying noise reduction measures. In this paper Wall-Modelled Large Eddy Simulations (WMLES), combined with the Ffowes Williams and Hawkings (FW-H) sound extrapolation method, are performed to reproduce three experimental cases, with the aim of isolating the different contributions of flap angle and trailing-edge/jet-axis distance $h$. The first case (DOAK), consisting of a single jet installed near a horizontal flat plate, confirms the fundamental mechanisms of jet-surface interaction and jet-surface reflection in the absence of lift. The second case (DLR-F16), with a coaxial jet installed under a high-lift wing, reveals the trailing-edge/jet-axis distance $h$ as the dominant parameter, with a possible influence of the flap angle at low frequencies. The third case (SYMPHONY) is used to study the interaction of a coaxial jet with a full aircraft geometry using Fourier decomposition of the pressure near-field to analyse the effects on sound sources and radiation.
\end{abstract}

\section{Nomenclature}

$a_{\infty} \quad$ speed of sound

$C$ airfoil chord length

$D_{b p}$ bypass diameter

$D_{j} \quad$ single-stream jet diameter

$f$ frequency

$h$ trailing-edge/jet-axis vertical distance

$l$ nozzle/trailing-edge axial distance

$M a \quad$ Mach number

$p \quad$ pressure

PSD power spectral density

$r$ radial coordinate

St Strouhal number

$t \quad$ physical time

$T$ temperature

$u \quad$ velocity
$U_{b p}$ bypass stream axial velocity

$U_{c} \quad$ core stream axial velocity

$U_{j} \quad$ single-stream jet axial velocity

$u_{\tau}$ friction velocity

$x \quad$ axial coordinate

$y \quad$ wall distance

$\varepsilon \quad$ upwinding parameter

$\theta$ polar angle

$v \quad$ viscosity

$v_{s g s}$ subgrid-scale viscosity

$v_{n w}$ near-wall turbulent viscosity

$\nu_{w} \quad$ wall turbulent viscosity

$\rho \quad$ density

$\sigma \quad$ singular value of the velocity gradient tensor

$\tau_{w} \quad$ wall shear stress

\footnotetext{
* Research Associate, Rolls-Royce University Technology Centre

${ }^{\dagger}$ Research Associate, Rolls-Royce University Technology Centre

$\ddagger$ PhD Student, Rolls-Royce University Technology Centre

${ }^{\S}$ Senior Lecturer, Rolls-Royce University Technology Centre

"IProfessor, Rolls-Royce University Technology Centre
} 


\section{Introduction}

The noise from a propulsive jet is an important source at take-off and approach conditions. In modern high bypass ratio turbofans there is an increased potential of interaction of the jet flow with the wing and the partially deployed high lift system, and its effect on noise need to be thoroughly investigated. Recent numerical studies [1-6] have shown that the combination of Large Eddy Simulation (LES) with the sound projection method of Ffowcs Williams and Hawkings (FW-H) [7] can successfully reproduce the installation effects. An adaptive Wall-Modelled LES introduced in a previous work [6], has proven its effectiveness and robustness. Its less stringent requirements of boundary refinement allow for a grid design focused on noise propagation, with low growth rates and low aspect ratios.

The aim of the present work is to apply this method, as described in Section $\Pi$, to study the sensitivity of noise generation to lift coefficient and to wing/engine relative positioning. Three experimental configurations have been identified to help isolate the different contributions. The first configuration (DOAK), consisting of a single stream jet in the vicinity of a semi-infinite flat plate, allows the study in the absence of lift, as discussed in Section IIII. The second configuration (DLR-F16), consisting of a coaxial jet under a three-element high-lift airfoil, is used in Section IV to assess the influence of flap parameters and jet/wing distance. The third configuration (SYMPHONY), presented in Section $\mathrm{V}$ extends the study to a full aircraft geometry, with wing, flap, pylon and fuselage.

\section{Numerical methods}

\section{A. Solver}

The solver [8] is a density-based cell-vertex finite volume industrial code originally developed for turbomachinery design. The second order flux calculation is based on the Roe scheme

$$
F_{i j}=\frac{1}{2}\left(F\left(Q_{i}\right)+F\left(Q_{j}\right)\right)-\frac{1}{2} \varepsilon\left|A_{i j}\right|\left(L_{j}(Q)-L_{i}(Q)\right)
$$

where $A_{i j}$ is the Jacobian of the inviscid flux, $Q$ is the conserved variable vector, $L()$ is the pseudo-Laplacian operator and $\varepsilon$ is a tunable parameter to control the amount of upwinding [9]. For the temporal discretisation, a standard three-stage Runge-Kutta explicit algorithm is employed.

\section{B. Adaptive Wall-Modelled LES}

The Wall-Modelled LES used in this work was validated in a previous study for this type of application [6]. It is based on the wall-stress modelling approach, in which the LES subgrid-scale model is defined all the way down to the wall, and the under-resolution of the boundary layer is compensated by imposing a wall-stress boundary condition.

\section{The subgrid-scale model}

The choice of the LES subgrid-scale (SGS) model is critical for jet applications. Standard models with constant coefficient are usually too dissipative in the initial free shear layer of the jet, thus delaying its transition. Dynamic models can overcome this issue [10], potentially at the price of stability, which is not always desirable when simulating complex flows. This is one reason why implicit LES (or ILES) has been widely adopted in the literature [9, 11, 12], since the absence of excessive SGS viscosity allows accurate capture of the jet behaviour, especially near the nozzle. Alternative SGS models [13, 14] to the original Smagorinsky model can yield a more physical behaviour for boundary and free shear layers, by replacing the classical strain rate in the formulation of the subgrid-scale viscosity $v_{S G S}$, with other invariants of the velocity field. One of the latest examples of this type of approach is the $\sigma$-model [15], which has been validated for jet noise [16, 17], and is chosen for the present work. The subgrid-scale viscosity is defined as

$$
v_{S G S}=\left(C_{\sigma} \Delta\right)^{2} \mathcal{D}_{\sigma}
$$

where $C_{\sigma}=1.35$ is the model constant, $\Delta$ is the subgrid characteristic length scale and

$$
\mathcal{D}_{\sigma}=\frac{\sigma_{3}\left(\sigma_{1}-\sigma_{2}\right)\left(\sigma_{2}-\sigma_{3}\right)}{\sigma_{1}^{2}}
$$

where $\sigma_{1} \geq \sigma_{2} \geq \sigma_{3} \geq 0$ are the three singular values of the velocity gradient tensor. Unlike more established models, such as Smagorinsky, the $\sigma$-model has, by construction, the appropriate cubic behaviour in the vicinity of solid 
boundaries without requiring any ad-hoc treatment. In addition, it has the property to automatically vanish as soon as the resolved field is two-dimensional, such as in pure shear, thus allowing for a faster development of the turbulent free shear layer of the jet, while still retaining the correct dissipation rate in fully developed turbulence further downstream [15]. The robustness of the model makes it a good candidate for complex applications.

\section{The wall shear stress boundary condition}

Following Werner and Wengle [18], the wall boundary condition is specified by assuming that at the grid points closest to the wall, the instantaneous velocity $u$ is in phase with the instantaneous wall shear stress $\tau_{w}$ and the instantaneous velocity distribution is assumed to follow the law-of-the-wall. Here we choose to use Spalding's continuous formula for the law-of-the-wall [19]

$$
y^{+}=u^{+}+\frac{1}{E}\left(e^{\kappa u^{+}}-1-\kappa u^{+}-\frac{\left(\kappa u^{+}\right)^{2}}{2 !}-\frac{\left(\kappa u^{+}\right)^{3}}{3 !}\right)
$$

where $y^{+}=y u_{\tau} / v, u^{+}=u / u_{\tau}, \kappa=0.41$ and $E=9.8$. The formula is solved iteratively for $u_{\tau}$ through the Newton-Raphson method. The resulting required wall shear stress

$$
\tau_{w}=\rho \cdot u_{\tau}^{2}
$$

is applied by defining the wall turbulent viscosity

$$
v_{w}=\frac{\tau_{w}}{\rho \cdot \partial u / \partial y}-v
$$

\section{The near-wall correction}

This wall-stress function needs to be balanced by an appropriate internal field in the immediate proximity of the wall. The under-resolution of the boundary layer means that the subgrid-scale viscosity, obtained with the $\sigma$-model, needs to be corrected near the wall, as it is zero by construction. The near-wall turbulent viscosity is therefore defined in a RANS fashion:

$$
v_{n w}=(\kappa y)^{2} \cdot\left(1-e^{-\left(y^{+} / A^{+}\right)^{3}}\right) \cdot S
$$

with $S=\sqrt{2 S_{i j} S_{i j}}, \kappa=0.41$ and $A^{+}=25$. This near-wall correction is necessary only in the first cell/node from the wall, since the LES model can quickly resume the LES behaviour in the adjacent layers. It is also an adaptive correction that can be used seamlessly with any resolution of the boundary layer (provided that the grid is relatively isotropic and the modelling only involves the inner boundary layer), since it vanishes with a physical behaviour when the boundary layer is fully resolved. This allows for a simple and robust implementation, with no additional parameters controlling the RANS-LES blending, as is required in DES or typical Hybrid RANS-LES models.

\section{Far-field acoustic computation}

For the far-field noise, the FW-H surface integral [20] is computed. This yields the far-field acoustic pressure fluctuation $p^{\prime}(\mathbf{x}, t)$. Since the noise source is inside the surface (if the surface is large enough and far enough from the jet exit region), a simplification can be made by omitting the volume quadrupole integral. This, as suggested by Shur et al. [21] and Di Francescantonio [20], saves substantial data storage. The integral equation is as follows:

$$
4 \pi p^{\prime}=\frac{\partial}{\partial t} \int_{S}\left[\frac{\rho u_{n}}{r}\right] d S+\frac{1}{a_{\infty}} \frac{\partial}{\partial t} \int_{S}\left[\frac{p_{n r}^{\prime}+\rho u_{n} u_{r}}{r}\right] d S+\int_{S}\left[\frac{p_{n r}^{\prime}+\rho u_{n} u_{r}}{r^{2}}\right] d S
$$

In the above, $\mathbf{r}$ ( $r$ being its modulus) defines the observer position, $a_{\infty}$ stands for the ambient speed of sound and $S$ is the FW-H surface. The quantities in the square brackets are computed at "retarded" times. Also, $n_{j}$ is the component of the unit outward normal vector on the surface, and $u_{j}$ is the velocity component. Surface data is stored while the simulation is performed, ready for later post-processing. 


\section{Single jet / flat plate interaction}

In order to analyse the impact of installation effects on the acoustic far-field in the absence of a flight stream, the noise produced by a single stream, isothermal jet, under static ambient flow conditions is studied with and without a horizontal semi-infinite flat plate installed close to the nozzle. According to Head and Fisher [22], there are two different mechanism in which the noise of the isolated nozzle is modified by the presence of the flat plate. The first mechanism is called jet-surface interaction (JSI) effect and is the dominant source of noise at low-frequencies. The JSI effect is produced by the interaction of the near-field acoustic pressure of the jet with the trailing-edge of the plate, which generates a dipole-type acoustic source. The second mechanism is known as jet-surface reflection (JSR) effect and it was discovered by Ffwocs-Williams and Hall [23]. The noise produced by this mechanism comes from the reflection of jet mixing noise by the bottom surface of the plate.

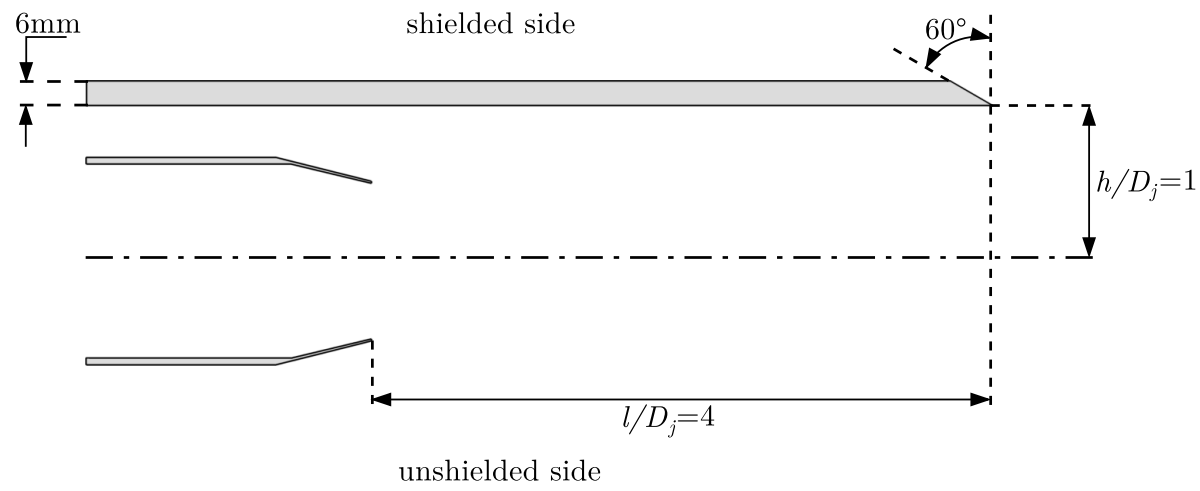

Fig. 1 Schematic of the DOAK geometry [24].

The geometry of the nozzle and flat plate in this study is based on the experiments carried out at the Doak Laboratory (DOAK) at the Institute of Sound and Vibration Research (ISVR) at the University of Southampton [24, 25]. The results presented in this work correspond to the radial location of the plate $h / D_{j}=1$ and axial extension $l / D_{j}=4$ (see Figure 1). The acoustic Mach number of the simulations is $M a=0.75$. Based on the diameter of the nozzle $(38.1 \mathrm{~mm})$ the Reynolds number of the cases is approximately $R e \approx 700,000$.

Results of the isolated and installed configurations are compared. The hexahedral grids are generated to be as similar as possible. Figure 2 presents a cut-plane of both grids at the nozzle exit. The total number of grid elements is $18 \mathrm{M}$ for the isolated configuration and $16 \mathrm{M}$ for the installed configuration. The spatial numerical scheme uses a smoothing parameter $\varepsilon=0.35$, which is considered a good compromise in terms of reducing the dissipation while avoiding spurious sound waves. $\varepsilon=1$ is used as a sponge zone near the far-field boundaries. The simulation was run using explicit time stepping ensuring CFL $<0.75$. A transient of $250 t^{*}$ (where the non-dimensional time $t^{*}=D_{j} / U_{j}$ ) was necessary to initialize the flow, and another $100 t^{*}$ was used for flow statistics and FW-H.

Figure 3 shows contours of the mean axial velocity for the isolated and the installed simulations. As expected, the isolated configuration presents a symmetry axis along the centreline (black dashed line). Conversely, in the installed configuration there is a slight asymmetry of the contour between the upper and lower sections of the dashed line. The asymmetry is produced by a small acceleration of the jet plume towards the plate due to a Coanda-type effect. Despite the difference in the symmetry of the jet plume, the contours of the two configurations are almost identical with similar shear layer growth and thickness, and potential core length.

The effect of the installation of the flat plate in the pressure field is depicted in Figure 4 A dipole-type acoustic source is visible at the trailing edge of the plate, generating low-frequency noise that modifies the acoustic field when comparing it to the isolated configuration. Since these waves are not generated near a high jet velocity region, their propagation does not suffer from refraction or Doppler effects and they propagate in an almost spherical manner. Furthermore, more energetic low-frequency waves are emitted in comparison with the isolated configuration. This is a first evidence of the jet-surface interaction (JSI) effect on the acoustic field. On the other hand, high-frequency waves are less visible on the shielded side of the plate due to the jet-surface reflection (JSR) mechanism.

To further analyse the installation effects on the noise prediction, the FW-H method is used to obtain the acoustic pressure signal at an observer location of $90^{\circ}$ and $r / D_{j}=53$. The coordinate system of the FW-H farfield observer positions is represented in Figure 5 and the location of the $\mathrm{FW}-\mathrm{H}$ surfaces for both configurations is shown in Figure 6 . The surface of the installed configuration follows the definition of the isolated simulation in the 

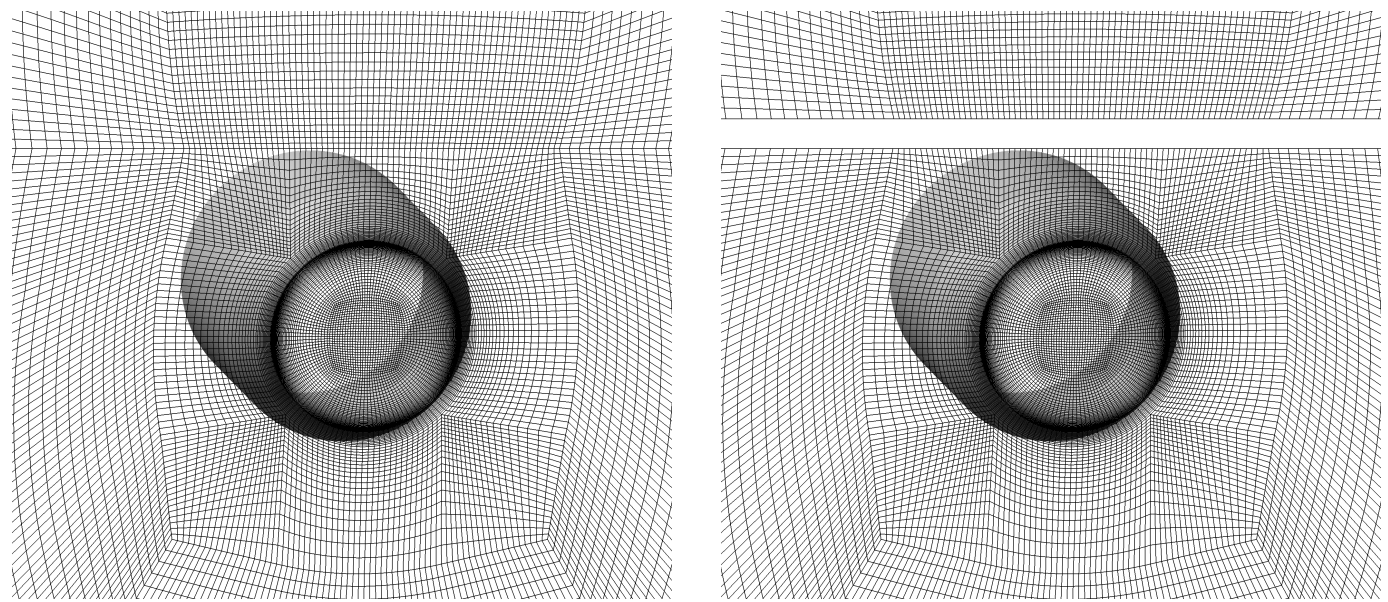

Fig. 2 Cut-plane of the mesh at the nozzle exit for the isolated (left) and installed (right) configuration.
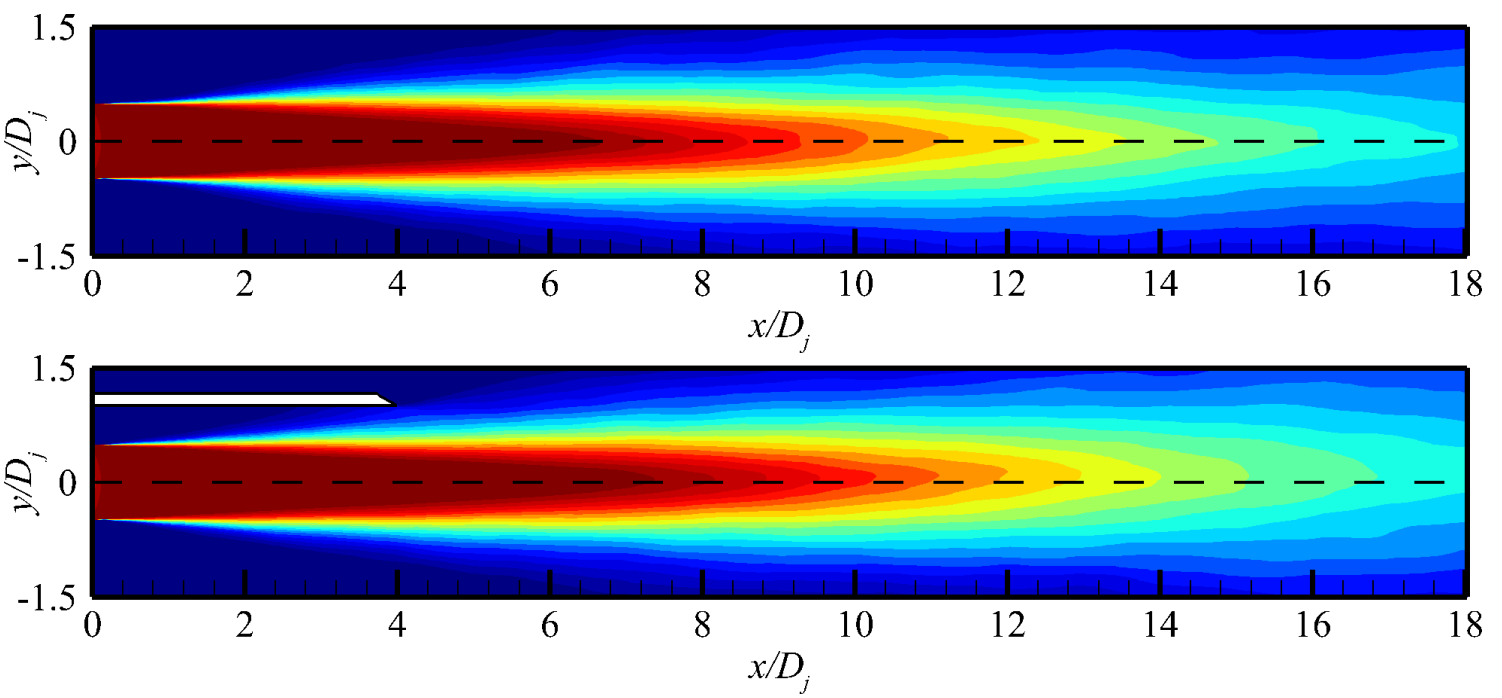

Fig. 3 Mean axial velocity contour for the isolated (top) and installed (bottom) configurations.
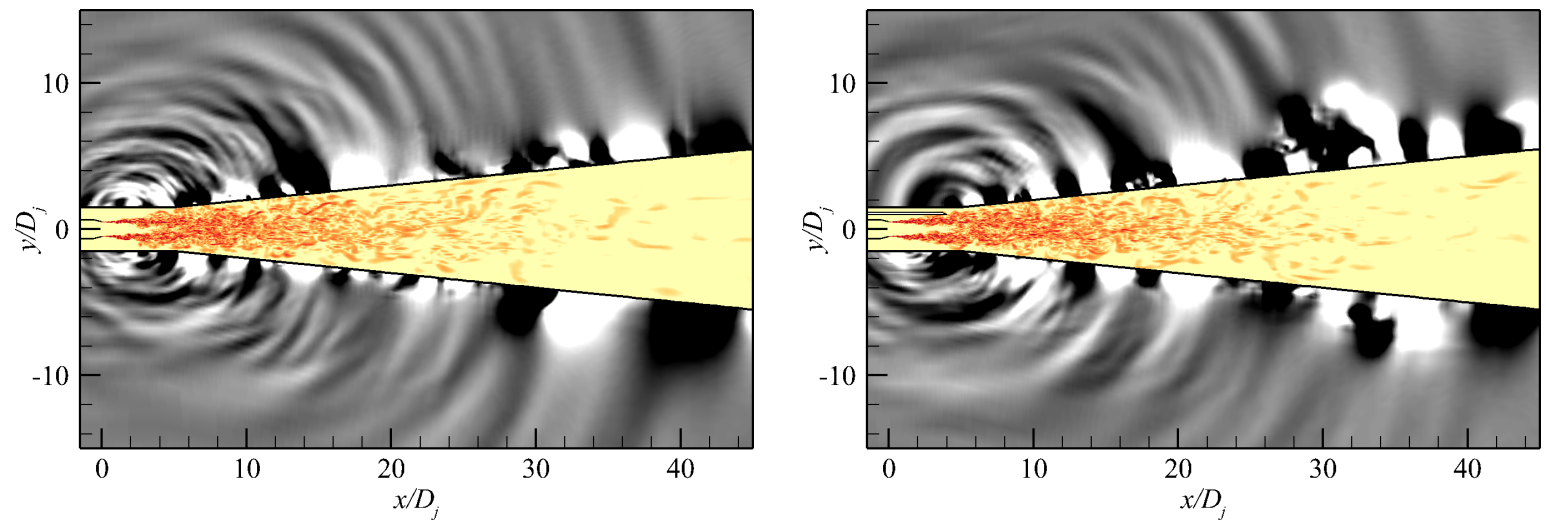

Fig. 4 Pressure perturbation with superimposed vorticity magnitude contours for the isolated (left) and installed (right) configurations. 
interval $-0.5 \leq y / D_{j} \leq 0, x / D_{j} \geq 4$ and it is stretched to include the contribution of the flat plate in the interval $0 \leq y / D_{j} \leq 1.5, x / D_{j} \leq 4$. To be able to evaluate the JSI and JSR effects, two different azimuthal angles have been studied. One corresponds to an observer below the plate, or in the unshielded side. The other one is located above the plate, or in the shielded side. Since the reflection of the waves only occurs in the unshielded side, the JSR effect should only be noticeable by the unshielded observer.

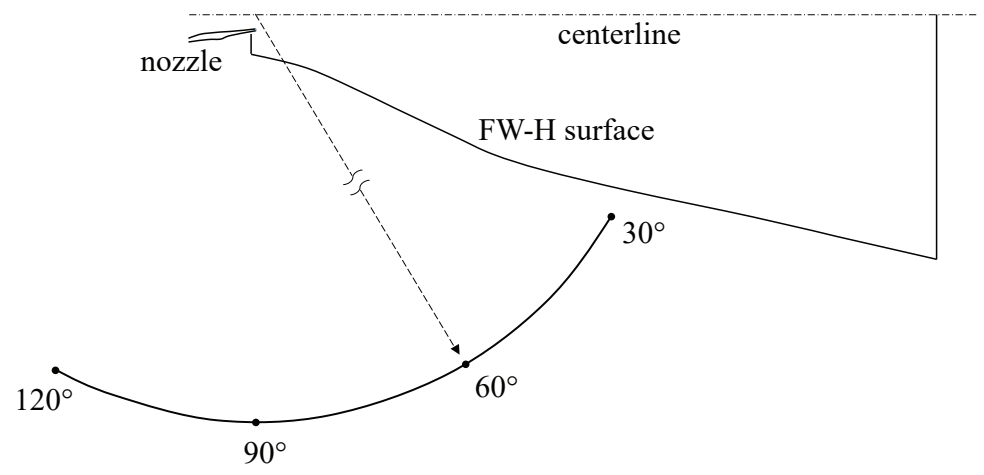

Fig. 5 Coordinate system of the FW-H far-field sound observer positions.
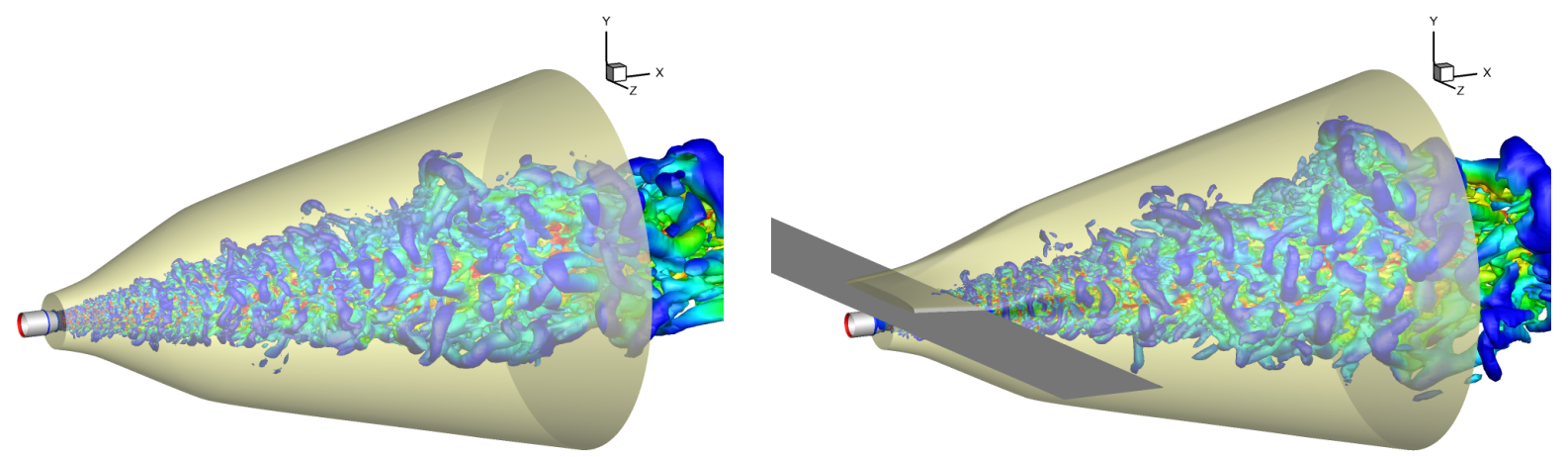

Fig. 6 FW-H surface location (in yellow) for the isolated (left) and installed (right) configurations. Q-criterion iso-surfaces coloured by axial velocity.

Power spectral density (PSD) plots, filtered by averaging over one-third octave bands, are presented in Figure 7 for both the unshielded and shielded sides. Until $S t=0.8$ both graphs show the same trend. The JSI effect is clearly visible with an increase of approximately $6 \mathrm{~dB}$ over the isolated configuration for the unshielded side and of $4 \mathrm{db}$ for the shielded side. For frequencies beyond $S t=0.8$, the two plots show a different behaviour. On the unshielded side, the PSD difference $\triangle \mathrm{PSD}=\mathrm{PSD}_{\text {inst }}-\mathrm{PSD}_{\text {isol }}$ is approximately $1 \mathrm{~dB}$. On the contrary, on the shielded side the value of $\triangle \mathrm{PSD}$ becomes negative. Therefore, the noise levels at higher frequencies of the isolated configuration are greater than in the installed one for observers in the shielded side. This is an evidence of the JSR mechanism, with the plate blocking the high-frequency sources generated in the jet plume and reflecting them towards the lower part of the plate.

\section{Coaxial jet / high-lift wing interaction}

In order to study the effects of flight stream on jet-surface interaction, and its dependence on flap parameters and jet/wing distance, numerical simulations are performed of the experiments carried out at the DLR Aeroacoustic Windtunnel Braunschweig (AWB) on the F16 straight high-lift-wing with an Ultra High Bypass Ratio (UHBR) nozzle [26]. In a limited span in the vicinity of the engine, the leading edge had no slat deployed in order to avoid intersection with the engine. A schematic of a cut-plane of the geometry is depicted in Figure 8. Approach flight conditions are imposed with a freestream velocity of $50 \mathrm{~m} / \mathrm{s}$ and a jet mixed Mach number of 0.37 . 


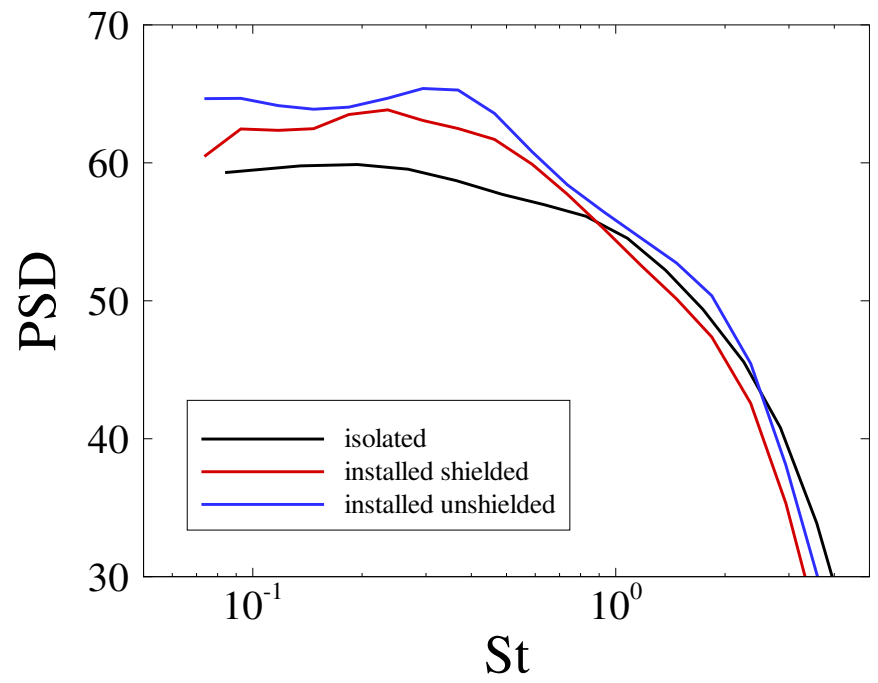

Fig. 7 Far-field sound PSD at polar angle $90^{\circ}$ for the isolated and installed configurations, on the unshielded and shielded side.

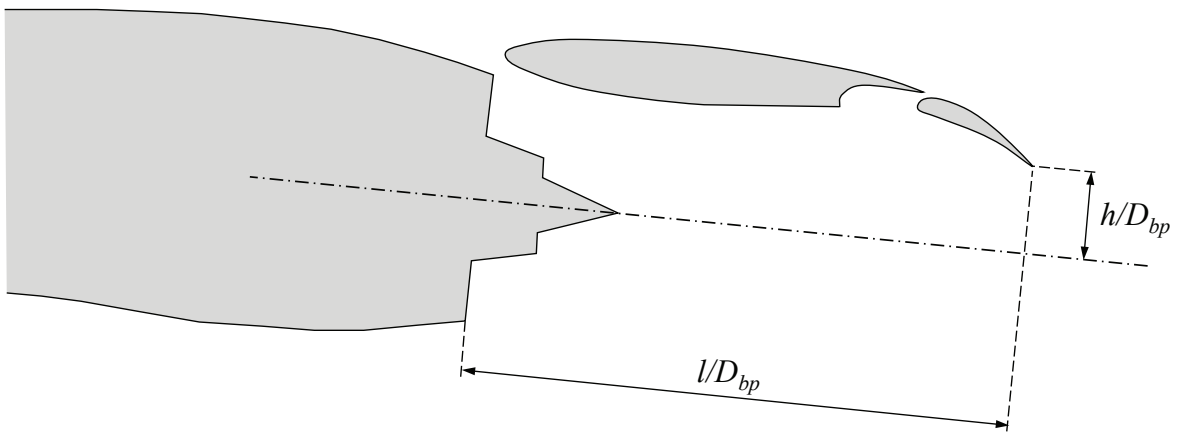

Fig. 8 Schematic of the DLR-F16 case. 


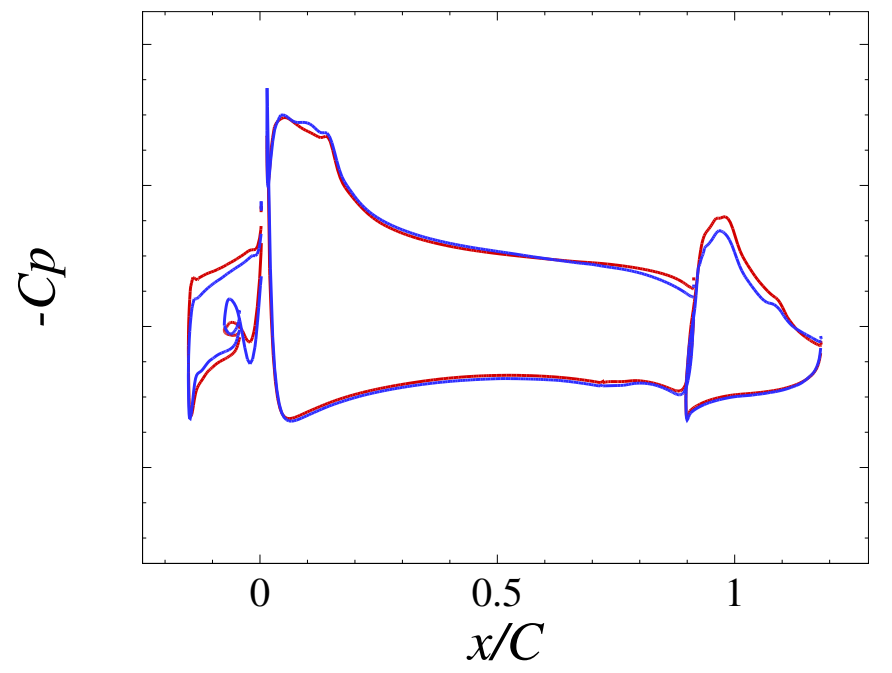

Fig. 9 Pressure coefficient distribution from RANS 2D simulations of the F16 wing. "Wind tunnel" freestream with $\mathrm{AoA}=14.5^{\circ}$ (red), and "infinite" freestream with $\mathrm{AoA}=6^{\circ}$ (blue).

In the experiments, wing and engine were mounted at an incidence angle of $14.5^{\circ}$ to take into account the limited cross-section of the wind tunnel flow. This angle was chosen to be equivalent to $6^{\circ}$ in an "infinite" freestream. A series of RANS simulations were performed for the present work in order to confirm this wind tunnel correction. Figure 9 shows the good agreement between the plots of pressure coefficient distribution along the wing in a "wind tunnel" freestream with $\mathrm{AoA}=14.5^{\circ}$ and in an "infinite" freestream with $\mathrm{AoA}=6^{\circ}$.

The Large Eddy Simulations presented in this section were therefore conducted on the simpler "infinite"-freestream configuration. The jet flow is supplied by a feed pipe as shown in Figure 10. The computational domain ranges from $x / D_{b p}=-20$ to 60 in the axial direction, with a radial extent of $40 D_{b p}$. The $120 \mathrm{M}$ grid is almost completely structured in the areas of interest. The rest of the domain is unstructured and allows for a more rapid coarsening towards the far-field.

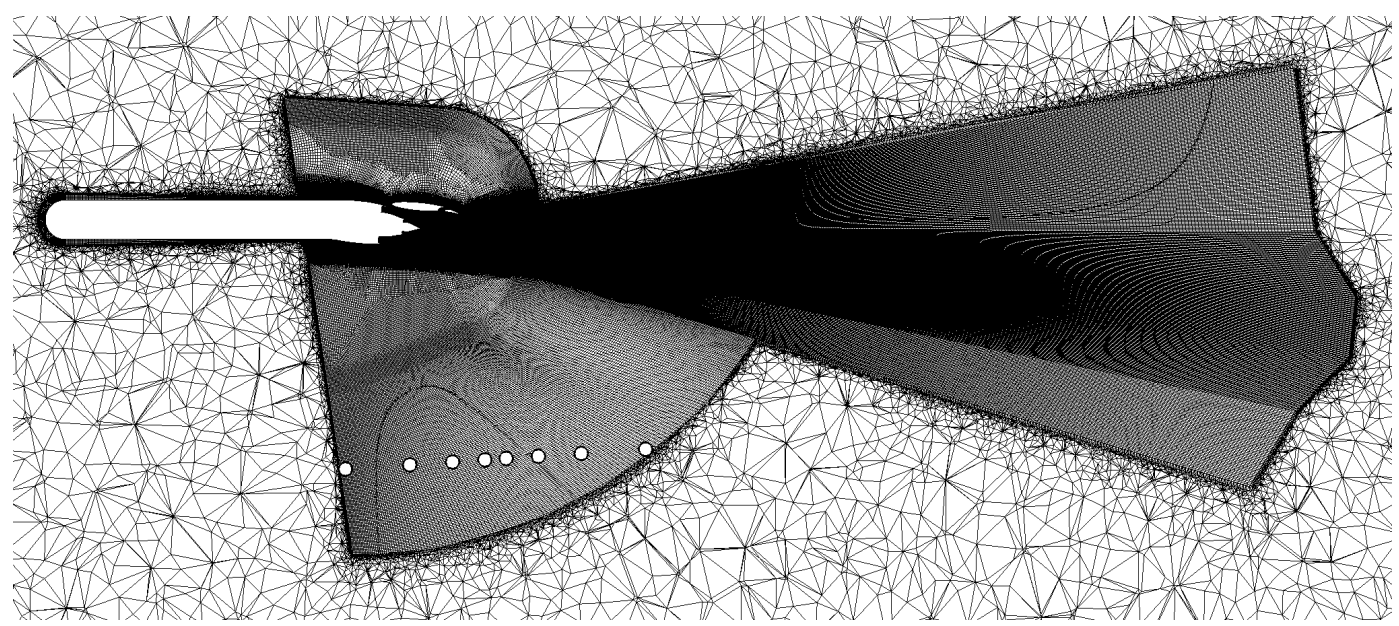

Fig. 10 Cut-plane of the DLR-F16 mesh. The white circles are the microphones locations. 
The spatial numerical scheme uses a smoothing parameter $\varepsilon=0.5$ to ensure stability. $\varepsilon=1$ is used as a sponge zone near the far-field boundaries. The simulation was run using explicit time stepping ensuring $\mathrm{CFL}<0.5$. A transient of $100 t^{*}$ (where the non-dimensional time $t^{*}=D_{b p} / U_{c}$ ) was necessary to initialize the flow, and another $50 t^{*}$ was used for flow statistics and near-field probe sampling.

Three configurations are studied, one with flap angle $14^{\circ}\left(h / D_{b p}=0.5\right)$ and two with flap angle $25^{\circ}$ but different wing/engine distance $\left(h / D_{b p}=0.43\right.$ and $\left.h / D_{b p}=0.59\right)$. This will allow to determine the dominant factor between flap angle and $h / D_{b p}$. The effectiveness of the wall modelling is confirmed by comparing the pressure coefficient distribution with the experimental data. Figure 11 shows very good agreement for the first configuration, with the only discrepancy on the pressure side of the flap, where the jet impinges the solid boundary in the simulation, while is presumed to be off in the experimental data. Similar plots were obtained for the other two configurations.

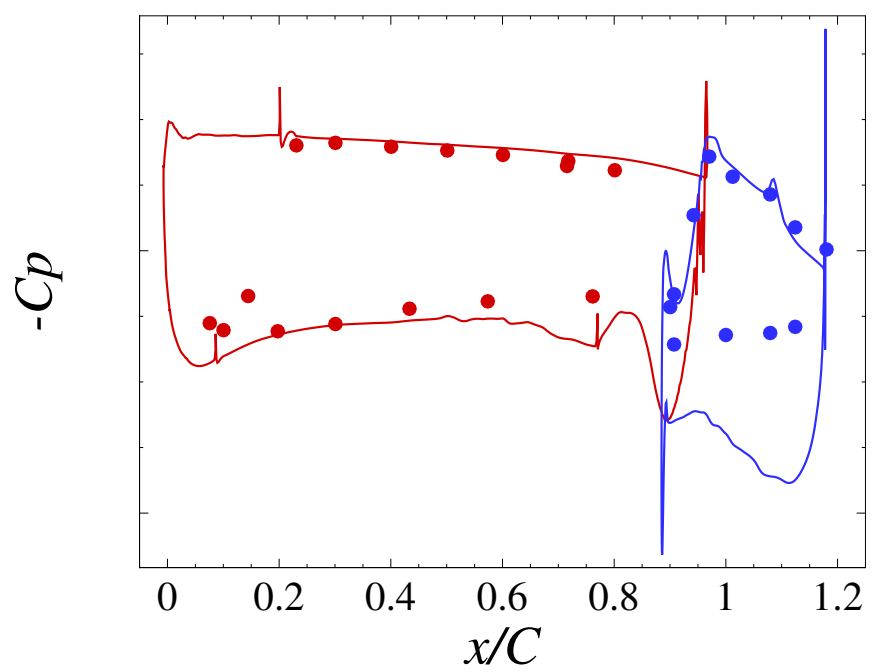

Fig. 11 Pressure coefficient distribution of the main body (red) and flap (blue) of the DLR-F16 case with flap angle $14^{\circ}$. The dots are from experiments, the solid line from LES.

The velocity contours in Figure 12 for the three configurations provide a very clear representation of the different jet/flap interaction. It is evident that the second configuration, with flap angle $25^{\circ}$ and $h / D_{b p}=0.43$, generates the strongest deflection of the jet. Similarly, the contours on a cut-plane at $x / D_{b p}=4$ in Figure 13 show how in the second configuration the jet is not only deflected, but its cross section is transformed by the interaction with the flap trailing edge into a more rectangular shape with a clear flat top side, whereas in the other two configurations the shape remains mostly circular.

The acoustic analysis for this case was conducted by introducing numerical probes in the near field of the jet, positioned in the same locations of the experimental microphones, as shown in Figure 10, and named channel 1 to 8 from left to right. A particular effort has been made to ensure low growth rates and low aspect ratios of grid cells, for an optimal noise propagation to the numerical probes. Given the relative proximity of the microphones, channels 1 and 4 were chosen as representative of the set.

Figure 14 shows the power spectral density (PSD) plots, filtered by averaging over one-third octave bands. Both channels 1 and 4 present an increase in peak noise of 2-4dB from the first configuration (flap angle $14^{\circ}, h / D_{b p}=0.5$ ) to the second (flap angle $25^{\circ}, h / D_{b p}=0.43$ ). The third configuration (flap angle $25^{\circ}, h / D_{b p}=0.59$ ) has a similar spectrum to the first from channel 1 . This seems to indicate that the trailing-edge/jet-axis distance $h$ is the dominant parameter controlling the installation noise. However, in channel 4 the third configuration presents a noise reduction, which can be attributed to the longer distance $h$, only for frequencies higher than $\mathrm{St} \approx 0.7$; for lower frequencies, the second and third configurations show a similar sound level. This trend seems to suggest that the low frequency content could also depend on pure trailing-edge noise, and therefore be influenced by the flap angle as well as by $h$, but a longer time sampling will be required to confirm this low-frequency effect. 


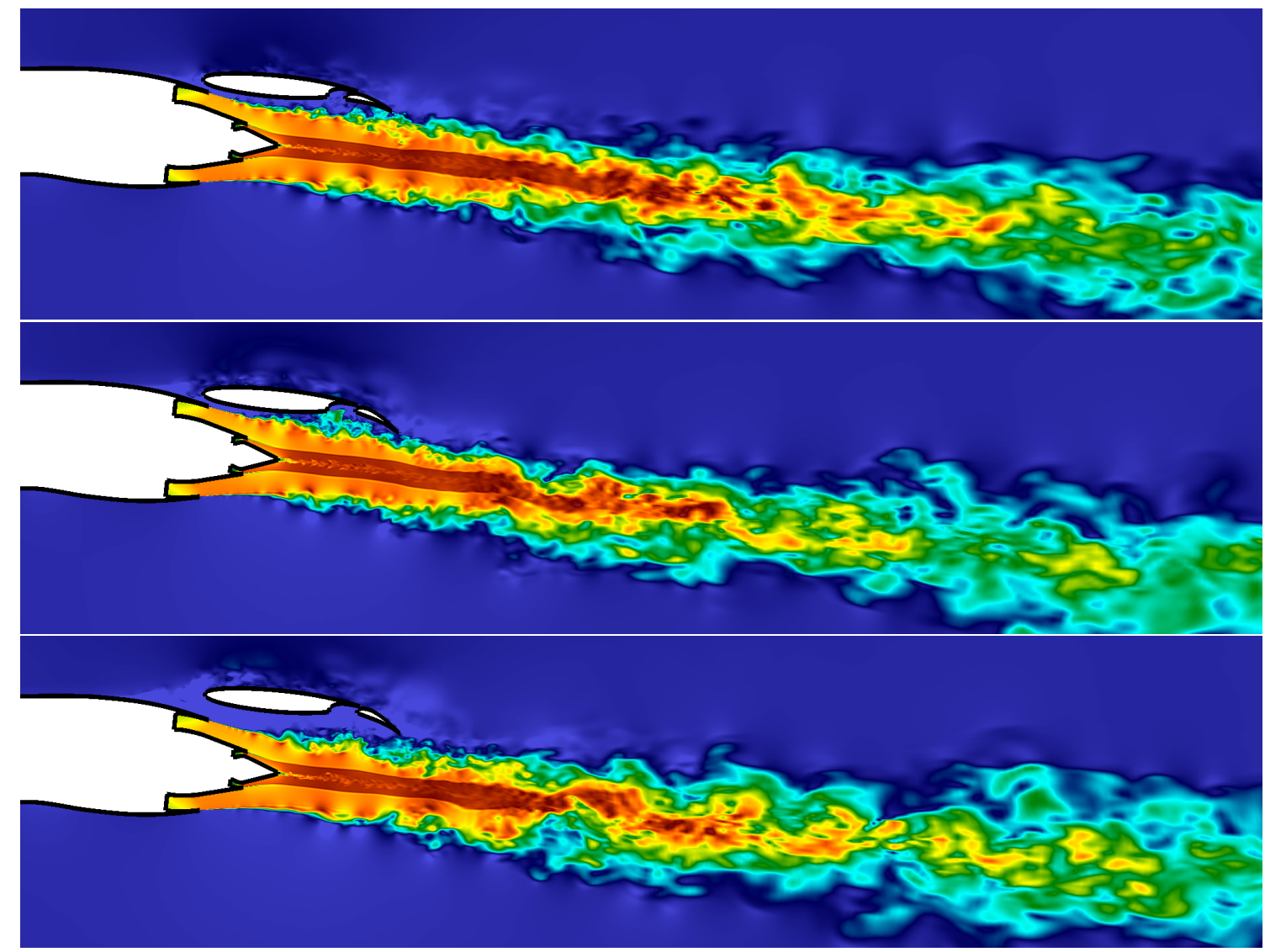

Fig. 12 Velocity contours on a cut plane at $y / D_{b p}=0$ for flap angle $14^{\circ}$ with $h / D_{b p}=0.5$ (top), flap angle $25^{\circ}$ with $h / D_{b p}=0.43$ (centre), and flap angle $25^{\circ}$ with $h / D_{b p}=0.59$ (bottom).
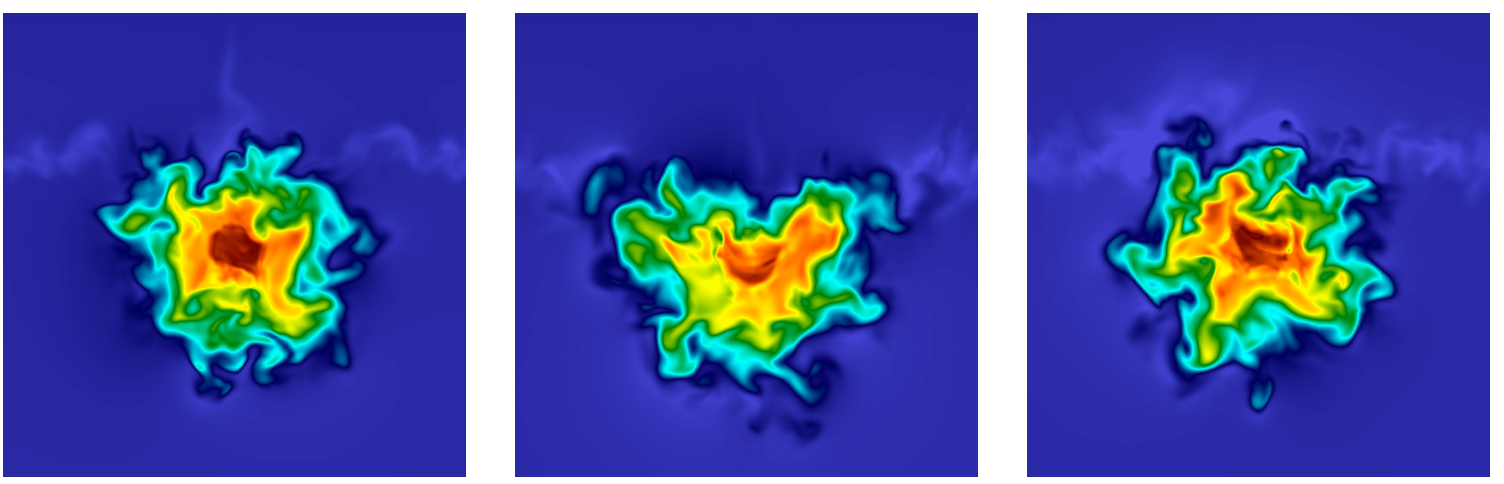

Fig. 13 Velocity contours on a cut plane at $x / D_{b p}=4$ for flap angle $14^{\circ}$ with $h / D_{b p}=0.5$ (left), flap angle $25^{\circ}$ with $h / D_{b p}=0.43$ (centre), and flap angle $25^{\circ}$ with $h / D_{b p}=0.59$ (right). 

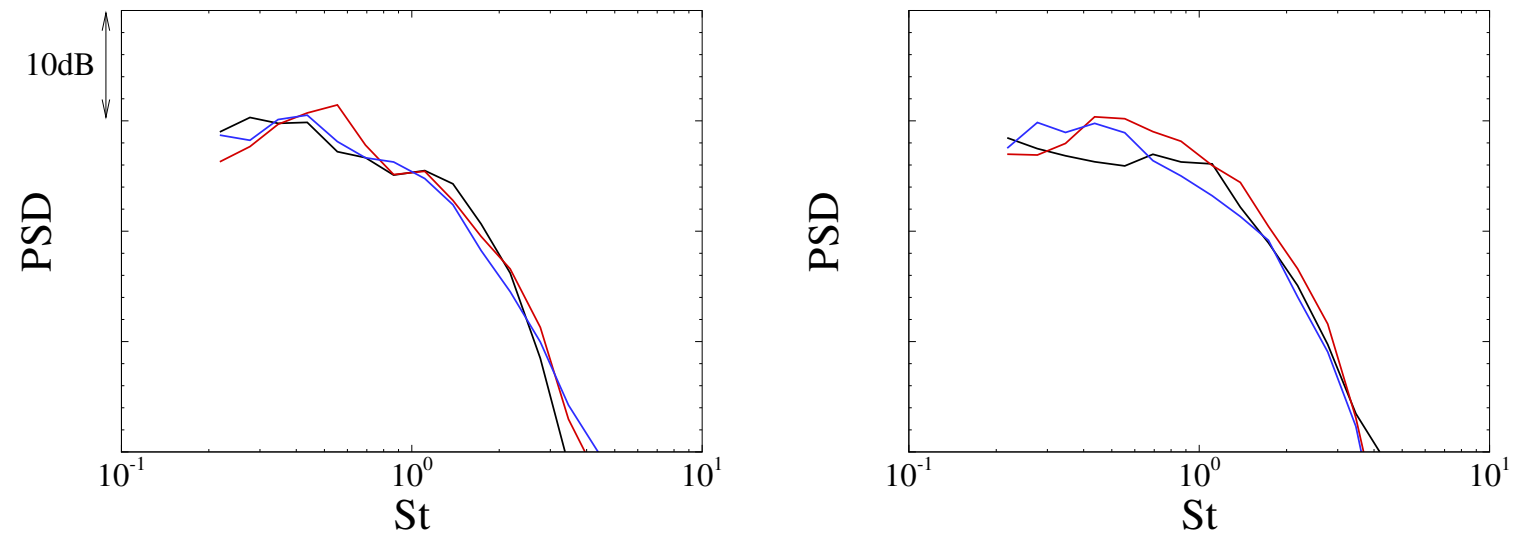

Fig. 14 Near-field sound PSD for microphones 1 (left) and 4 (right) for flap angle $14^{\circ}$ with $h / D_{b p}=0.5$ (black), flap angle $25^{\circ}$ with $h / D_{b p}=0.43$ (red), and flap angle $25^{\circ}$ with $h / D_{b p}=0.59$ (blue).

\section{Coaxial jet / aircraft interaction}

The final case consists of a simplified transport aircraft geometry, with fuselage, one wing with flaps deployed, and the coaxial engine nozzle with pylon. Geometry and experimental data were produced under the SYMPHONY project. The flow conditions are representative of the range of conditions encountered by modern aeroengines in post-takeoff. For additional details on the computational domain and the $120 \mathrm{M}$ grid, as well as on the FW-H procedure, the reader is referred to Angelino et al. [6]. The spatial numerical scheme uses a smoothing parameter $\varepsilon=0.5$. $\varepsilon=1$ is used as a sponge zone near the far-field boundaries. The simulation was run using explicit time stepping ensuring $\mathrm{CFL}<0.5$. A transient of $200 t^{*}$ (where the non-dimensional time $t^{*}=D_{b p} / U_{c}$ ) was necessary to initialize the flow, and another $150 t^{*}$ was used for flow statistics and FW-H. Preliminary results [6] showed good agreement with experiments for flap angle $16^{\circ}\left(h / D_{b p}=0.51\right)$. Here an additional configuration is investigated, with flap angle $32^{\circ}\left(h / D_{b p}=0.36\right)$.

Figure 15 shows the jet Q-criterion, and acoustic waves on a cut-plane immediately below the engine, for the two configurations. The $32^{\circ}$ configuration presents a slightly stronger pressure footprint, and a higher deflection of the jet, as confirmed by the velocity contours of Figure 16. The higher flap angle is responsible for an increased interaction of the jet with the flap trailing edge.

In order to further study the effect of this increased interaction on jet noise, a Fourier analysis can be applied to the pressure field to isolate the contributions of single frequencies. The temporal Fourier transform of the pressure fluctuation $\hat{p}(f)=\int_{-\infty}^{+\infty} p(t) e^{-2 \pi f t} d t$ is filtered at Strouhal numbers $\mathrm{St}=0.3$ and 1.0, and the real part of its inverse
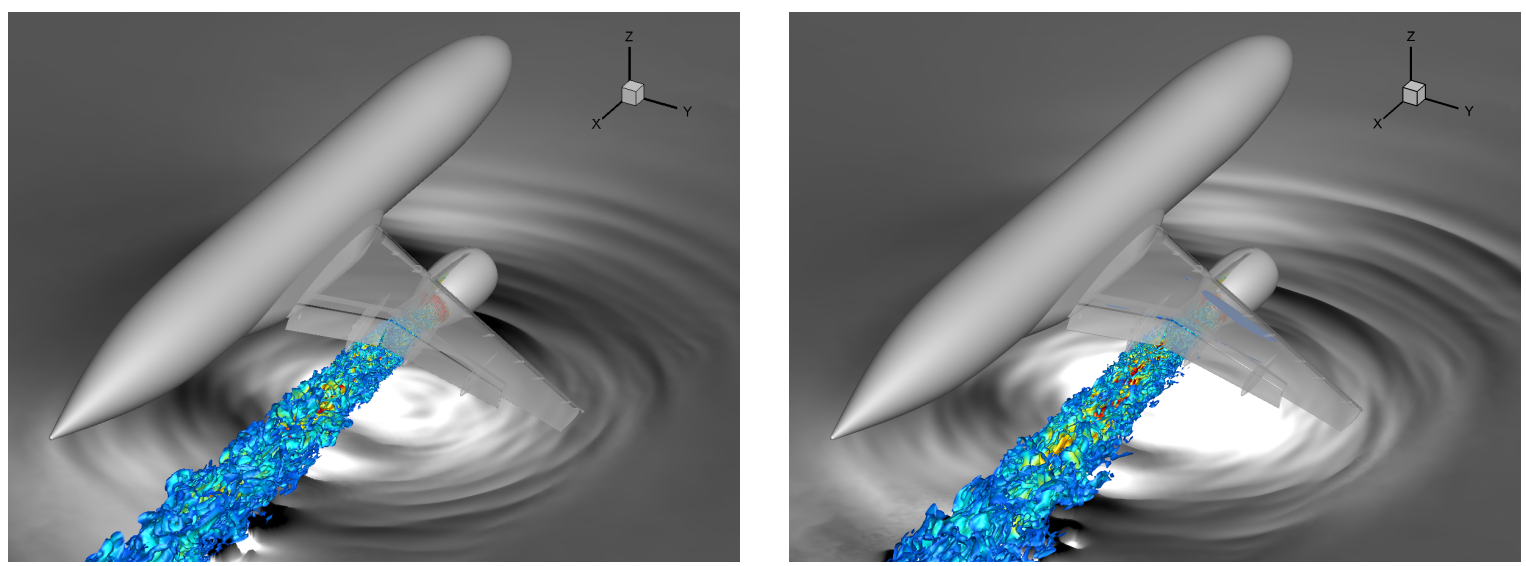

Fig. 15 Jet Q-criterion coloured by axial velocity (rainbow), and pressure waves at $z / D_{b p}=-0.2$ (greyscale). Flap angle $16^{\circ}$ on the left, $32^{\circ}$ on the right. The solid surfaces are representative of the experimental geometry. 

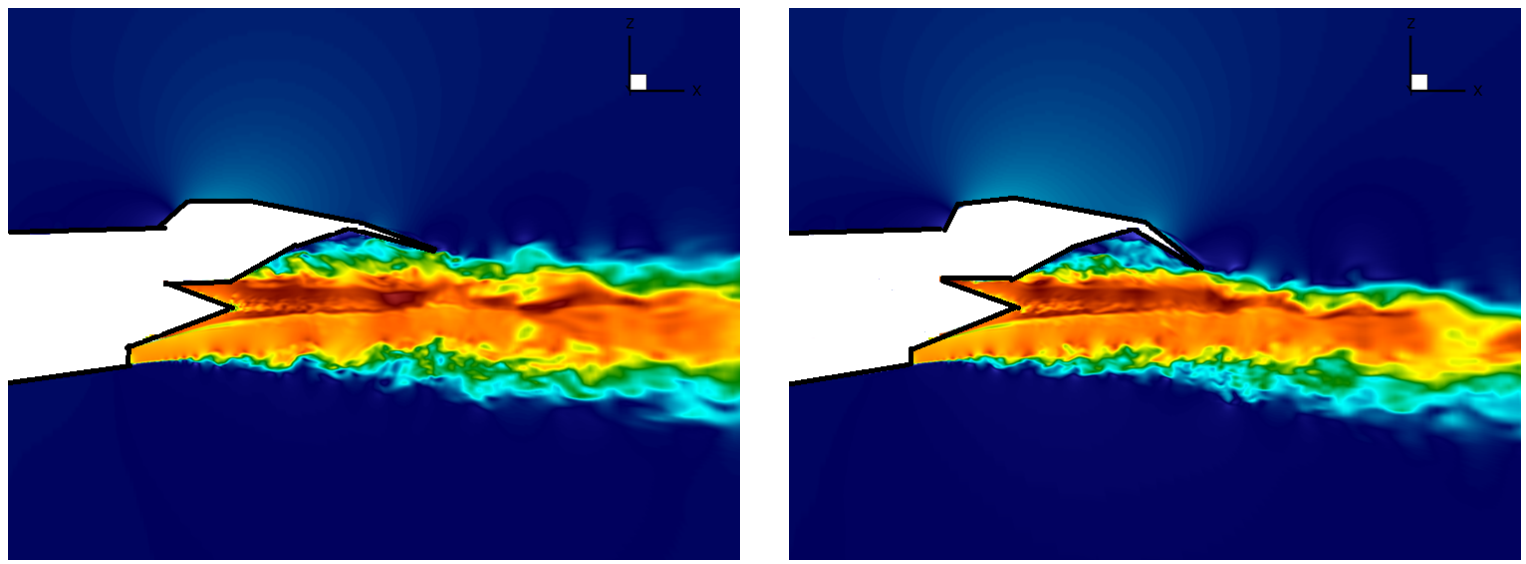

Fig. 16 Velocity contours with flap angle $16^{\circ}$ (left) and $32^{\circ}$ (right).
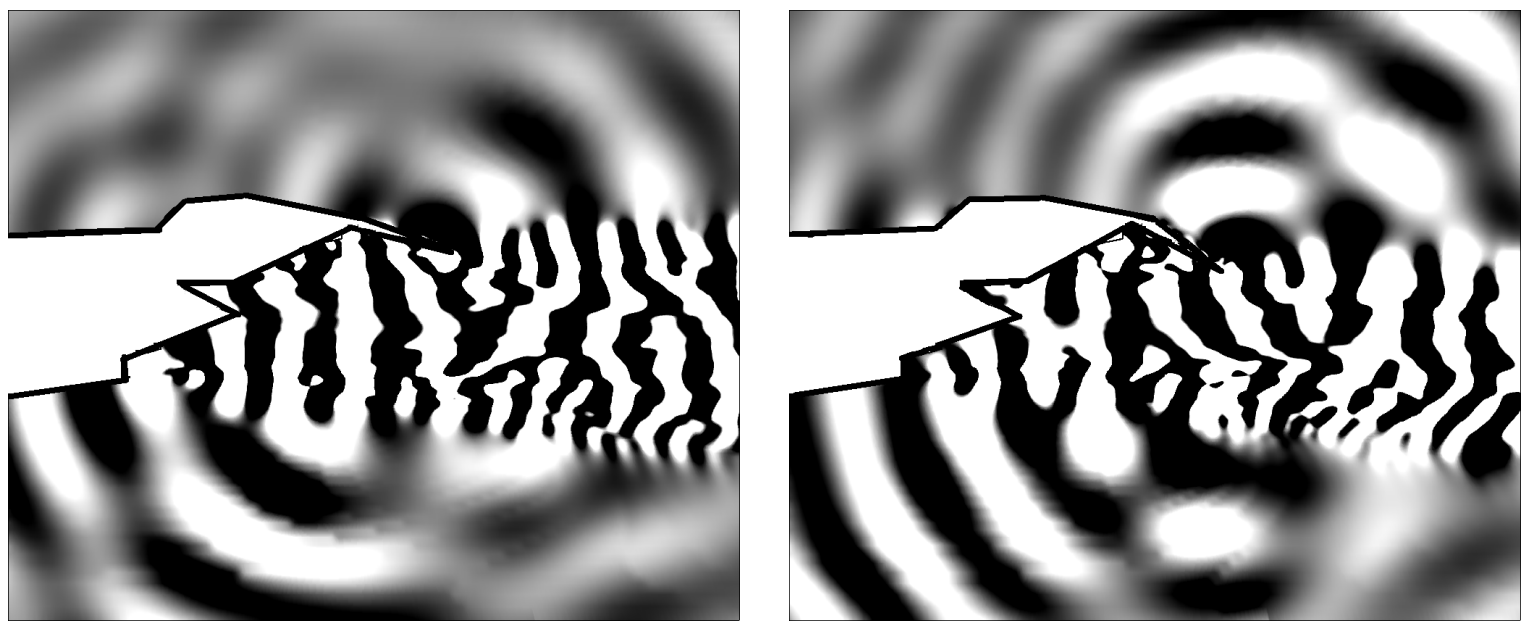

Fig. 17 Real part of the filtered inverse Fourier transform of pressure fluctuations for $\mathrm{St}=0.3$ with flap angle $16^{\circ}$ (left) and $32^{\circ}$ (right). Color scale from -3 to $3 \mathrm{~Pa}$.
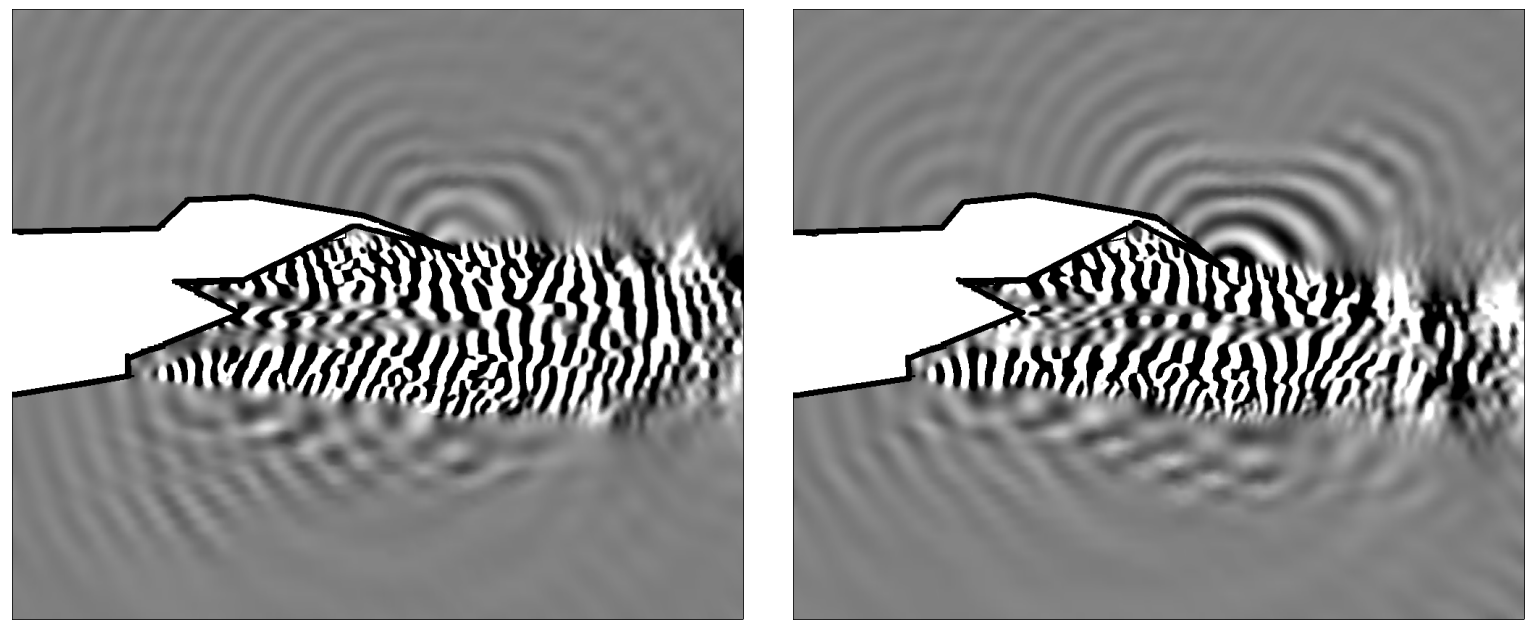

Fig. 18 Real part of the filtered inverse Fourier transform of pressure fluctuations for $\mathrm{St}=1.0$ with flap angle $16^{\circ}$ (left) and $32^{\circ}$ (right). Color scale from -3 to 3 Pa. 
transform is shown in Figure 17 and Figure 18 for the two flap angles. A dominant source of lower-frequency noise $(\mathrm{St}=0.3)$ can be located at the trailing edge of the flap, and the strength of the waves increases noticeably with the flap angle. At higher frequencies $(\mathrm{St}=1.0)$ sources spread more evenly along the whole mixing region, from the nozzle lip to beyond the end of the jet potential core. It is possible to notice a high-frequency scattering from the wing leading edge, and the expected noise source at the flap trailing edge, which again is stronger for the configuration with higher flap angle.

This qualitative analysis of the effect of the flap angle is confirmed by the FW-H far-field spectra in Figure 19 obtained for two "flyover" observer locations at polar angles $60^{\circ}$ and $120^{\circ}$ (see reference frame in Figure 5). As in the previous sections, the PSD is filtered by averaging over one-third octave bands. In both experimental and numerical results, the shift in noise level due the different flap angle is modest (2-4dB) but consistent, especially for St $>0.3$, which corroborates the results obtained in the Section IV
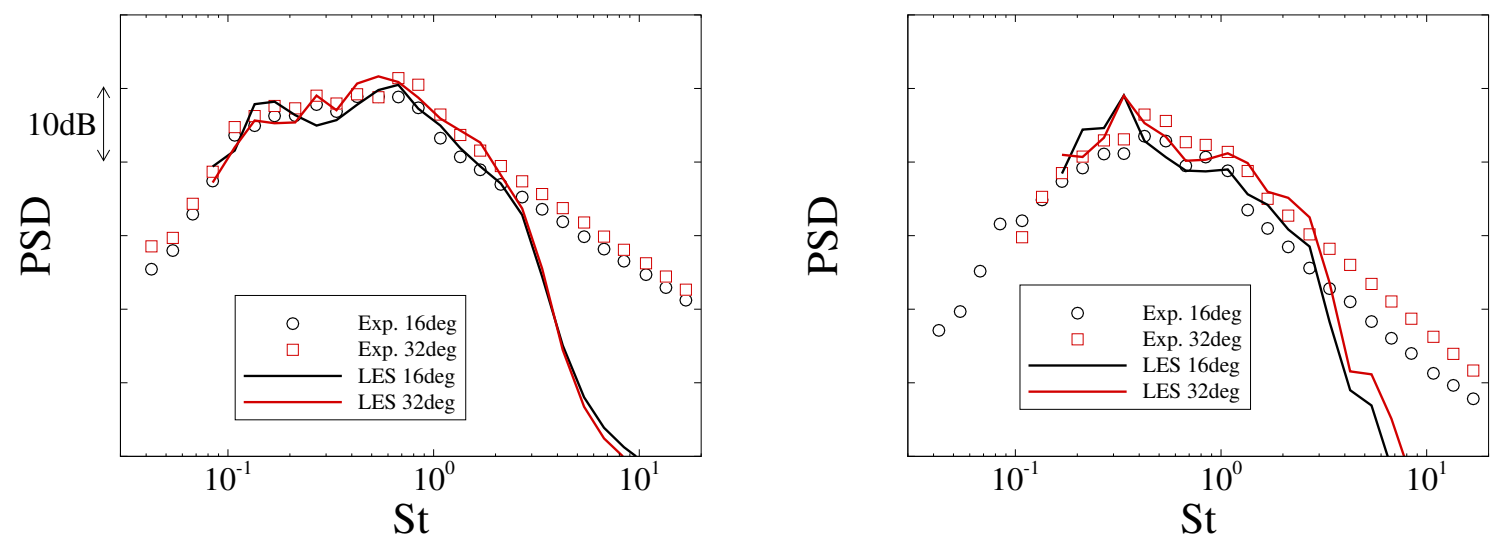

Fig. 19 Far-field sound PSD at polar angle $60^{\circ}$ (left) and $120^{\circ}$ (right).

\section{Conclusions}

Large eddy simulations of three different experimental cases were performed to show the effects on jet noise of lift and wing/engine positioning.

The first case (DOAK), consisting of a single jet installed near a horizontal flat plate, confirmed the effectiveness of the numerical method in capturing the fundamental mechanisms of jet-surface interaction (JSI) and jet-surface reflection (JSR). Pressure field contours and far-field sound spectra reveal a dipole-type acoustic source at the trailing edge of the plate generating strong low-frequency noise in all directions (JSI), as well as weakened high-frequency noise on the shielded side of the plate due to reflection (JSR). The configuration of the case, with no freestream (and therefore no lift) and zero incidence angle between jet and flat plate, provided a first indication of the importance of the trailing-edge/jet-axis distance $h$.

The second case (DLR-F16), with a coaxial jet installed under a high-lift wing, was studied for three different configurations, allowing for a direct comparison of the influence of $h / D_{b p}$ and flap angle. Velocity contours identify $h / D_{b p}$ as the dominant parameter affecting the deflection of the jet. Near-field sound spectra confirm a dependence on $h / D_{b p}$ especially in the high-frequency range, whereas low-frequency noise seems to be influenced by pure trailing-edge noise and therefore by the flap angle.

The third and final case (SYMPHONY) extended the study to the installation of a coaxial jet on a full aircraft geometry. The Fourier analysis of the noise spectral components reveals the combined effect of $h / D_{b p}$ and flap angle on the pressure field. The interaction of the jet with the flap produces a dominant source of low-frequency noise at the flap trailing edge, while higher-frequency sources spread more evenly along the whole mixing region. The enhanced jet/flap interaction due to lower $h / D_{b p}$ and higher flap angle has a broadband effect on the strength of these sources, as confirmed by the shift in noise level in the far-field noise spectra. 


\section{Acknowledgments}

This work is part of the ongoing Innovate UK ACAPELLA project (reference number 113086), in collaboration with Rolls-Royce plc. The geometry for the case presented in Section III was produced by the Institute of Sound and Vibration Research (ISVR) at the University of Southampton. Geometry and experimental data for the case presented in Section $[\mathrm{IV}$ were kindly provided by DLR. Geometry and experimental data for the case presented in Section $\mathrm{V}$ were produced under the Innovate UK SYMPHONY project (reference number 100539) and provided by Rolls-Royce plc.

The authors would like to thank EPSRC for the computational time made available via the UK Turbulence Consortium, under grant number EP/L000261. This work used the ARCHER UK National Supercomputing Service (http://www.archer.ac.uk). We acknowledge the use of Athena at HPC Midlands+, which was funded by the EPSRC on grant EP/P020232/1.

\section{References}

[1] Paliath, U., and Premasuthan, S., "Large Eddy Simulation for Jet Installation Effects," 2013. doi:10.2514/6.2013-2137.

[2] Mockett, C. R., Fuchs, M., Kramer, F., Michel, U., Steger, M., and Thiele, F., “Turbulence Modelling and Meshing Developments for the Prediction of Jet Noise Installation Effects," 2016. doi:10.2514/6.2016-2933.

[3] Tyacke, J. C., Wang, Z.-N., and Tucker, P. G., "LES-RANS of installed ultra-high bypass-ratio coaxial jet aeroacoustics with a finite span wing-flap geometry and flight stream - Part 1: round nozzle," 2017. doi:10.2514/6.2017-3854.

[4] Wang, Z.-N., Tyacke, J. C., and Tucker, P. G., "LES-RANS of installed ultra-high bypass-ratio coaxial jet aeroacoustics with a finite span wing-flap geometry and flight stream - Part 2: chevron nozzle," 2017. doi:10.2514/6.2017-3855.

[5] Gand, F., Huet, M., Le Garrec, T., and Cléro, F., "Jet noise of a UHBR nozzle using ZDES: external boundary layer thickness and installation effects," 2017. doi:10.2514/6.2017-3526.

[6] Angelino, M., Xia, H., and Page, G. J., "Adaptive Wall-Modelled Large Eddy Simulation of Jet Noise in Isolated and Installed Configurations," American Institute of Aeronautics and Astronautics, 2018. doi:10.2514/6.2018-3618.

[7] FFowcs Williams, J. E., and Hawkings, D. L., "Sound Generation by Turbulence and Surfaces in Arbitrary Motion,” Philosophical Transactions of the Royal Society A: Mathematical, Physical and Engineering Sciences, Vol. 264, No. 1151, 1969, pp. 321-342. doi:10.1098/rsta.1969.0031.

[8] Moinier, P., "Algorithm developments for an unstructured viscous flow solver," PhD thesis, University of Oxford, 1999.

[9] Xia, H., Tucker, P. G., and Eastwood, S., "Large-eddy simulations of chevron jet flows with noise predictions," International Journal of Heat and Fluid Flow, Vol. 30, No. 6, 2009, pp. 1067-1079. doi:10.1016/j.ijheatfluidflow.2009.05.002.

[10] Angelino, M., Boghi, A., and Gori, F., "Numerical solution of three-dimensional rectangular submerged jets with the evidence of the undisturbed region of flow," Numerical Heat Transfer, Part A: Applications, Vol. 70, No. 8, 2016, pp. 815-830. doi:10.1080/10407782.2016.1214494.

[11] Grinstein, F. F., and Fureby, C., "Recent Progress on MILES for High Reynolds Number Flows," Journal of Fluids Engineering, Vol. 124, No. 4, 2002, p. 848. doi:10.1115/1.1516576.

[12] Tucker, P. G., "Novel MILES computations for jet flows and noise," International Journal of Heat and Fluid Flow, Vol. 25, No. 4, 2004, pp. 625-635. doi:10.1016/j.ijheatfluidflow.2003.11.021.

[13] Nicoud, F., and Ducros, F., "Subgrid-Scale Stress Modelling Based on the Square of the Velocity Gradient Tensor," Flow, Turbulence and Combustion, Vol. 62, No. 3, 1999, pp. 183-200.

[14] Vreman, A. W., "An eddy-viscosity subgrid-scale model for turbulent shear flow: Algebraic theory and applications," Physics of Fluids, Vol. 16, No. 10, 2004, pp. 3670-3681. doi:10.1063/1.1785131.

[15] Nicoud, F., Toda, H. B., Cabrit, O., Bose, S., and Lee, J., "Using singular values to build a subgrid-scale model for large eddy simulations," Physics of Fluids, Vol. 23, No. 8, 2011, p. 085106. doi:10.1063/1.3623274.

[16] Angelino, M., Xia, H., Moratilla-Vega, M. A., and Page, G. J., "Far-field Noise Prediction of Round and Serrated Jets with Increasingly Refined Grids,” Lyon, France, 2016. doi:10.2514/6.2016-3047. 
[17] Angelino, M., Moratilla-Vega, M. A., Xia, H., and Page, G. J., "Large-eddy simulations of high Reynolds number jets with a suitable subgrid-scale model for solver dependency study," 11th International ERCOFTAC Symposium on Engineering Turbulence Modelling and Measurements, Palermo, Italy, 2016.

[18] Werner, H., and Wengle, H., "Large-Eddy Simulation of Turbulent Flow Over and Around a Cube in a Plate Channel," Turbulent Shear Flows 8, Springer Berlin Heidelberg, Berlin, Heidelberg, 1993, pp. 155-168.

[19] Spalding, D. B., "A Single Formula for the "Law of the Wall”," Journal of Applied Mechanics, Vol. 28, No. 3, 1961 , p. 455. doi:10.1115/1.3641728.

[20] Di Francescantonio, P., "A new boundary integral formulation for the prediction of sound radiation," Journal of Sound and Vibration, Vol. 202, No. 4, 1997, pp. 491-509. doi:10.1006/jsvi.1996.0843.

[21] Shur, M., Spalart, P., Strelets, M., and Travin, A., "Towards the prediction of noise from jet engines," International Journal of Heat and Fluid Flow, Vol. 24, No. 4, 2003, pp. 551-561. doi:10.1016/S0142-727X(03)00049-3.

[22] Head, R., and Fisher, M., "Jet/surface interaction noise - Analysis of farfield low frequency augmentations of jet noise due to the presence of a solid shield," 3rd Aeroacoustics Conference, American Institute of Aeronautics and Astronautics, Palo Alto,CA,U.S.A., 1976. doi:10.2514/6.1976-502.

[23] FFowcs Williams, J. E., and Hall, L. H., "Aerodynamic sound generation by turbulent flow in the vicinity of a scattering half plane," Journal of Fluid Mechanics, Vol. 40, No. 04, 1970, p. 657. doi:10.1017/S0022112070000368.

[24] Lawrence, J., "Aeroacoustic Interactions of Installed Subsonic Round Jets," PhD thesis, University of Southampton, 2014.

[25] Lawrence, J., Azarpeyvand, M., and Self, R., "Interaction between a Flat Plate and a Circular Subsonic Jet," 17th AIAA/CEAS Aeroacoustics Conference (32nd AIAA Aeroacoustics Conference), American Institute of Aeronautics and Astronautics, Portland, Oregon, 2011. doi:10.2514/6.2011-2745.

[26] Jente, C., Pott-Pollenske, M., Boenke, D., Buescher, A., and Goldhahn, I., "Experimental Investigation of Jet-Flap-Interaction Noise Sensitivity due to varying flap parameters at a UHBR Engine/High-Lift-Wing installation," American Institute of Aeronautics and Astronautics, 2018. doi:10.2514/6.2018-3788.

[27] Mendez, S., Shoeybi, M., Lele, S., and Moin, P., "On the use of the Ffowcs Williams-Hawkings equation to predict far-field jet noise from large-eddy simulations," International Journal of Aeroacoustics, Vol. 12, No. 1-2, 2013, pp. 1-20. doi:10.1260/1475-472X.12.1-2.1. 